\title{
An other uncertainty principle for the Hankel transform
}

\author{
Chirine Chettaoui \\ Département de Mathématiques et d'Informatique, \\ Institut national des sciences appliquées et de Thechnologie, \\ Centre Urbain Nord BP 676 - 1080 Tunis cedex, Tunisia. \\ chirine.chettaoui@insat.rnu.tn
}

\begin{abstract}
We use the Hausdorff-Young inequality for the Hankel transform to prove the uncertainly principle in terms of entropy. Next, we show that we can obtain the HeisenbergPauli-Weyl inequality related to the same Hankel transform.
\end{abstract}

\section{RESUMEN}

Usamos la desigualdad de Hausdorff-Young para la transformada de Hankel para probar el principio de incertidumbre en términos de la entropía. Además probamos que podemos obtener la desigualdad de Heisenberg-Pauli-Weyl relacionada con la misma transformada de Hankel.

Keywords and Phrases: Uncertainty principle, Hausdorff-Young inequality, entropy, Hankel transform

2010 AMS Mathematics Subject Classification: 43A32, 42B25. 


\section{Introduction:}

The uncertainly principles play an import role in harmonic analysis. They state that a function $f$ and its Fourier transform $\widehat{f}$ can not be simultaneously sharply localized in the sense that it is impossible for a nonzero function and its Fourier transform to be simultaneously small.

Many mathematical formulations of this fact can be found in $[2,5,6,11,16,17]$.

For a probability density function $f$ on $\mathbb{R}^{n}$, the entropy of $f$ is defined according to [18] by

$$
E(f)=-\int_{\mathbb{R}^{n}} f(x) \ln (f(x)) d x .
$$

The entropy $E(f)$ is closely related to quantum mechanics [4] and constitutes one of the important way to measure the concentration of $f$.

The uncertainly principle in terms of entropy consists to compare the entropy of $|\mathbf{f}|^{2}$ with that of $|\widehat{|f|}|^{2}$.

A first result has been given in [13], where Hirschman established a weak version of this uncertainly principle by showing that for every square integrable function $f$ on $\mathbb{R}^{n}$ with respect to the Lebesgue measure, such that $\|f\|_{2}=1$, we have

$$
E\left(|f|^{2}\right)+E\left(\mid \widehat{f}^{2}\right) \geqslant 0
$$

Later, in $[1,2]$, Beckner proved the following stronger inequality, that is for every square integrable function $f$ on $\mathbb{R}^{n} ;\|f\|_{2}=1$,

$$
E\left(|f|^{2}\right)+E\left(\mid \widehat{f}^{2}\right) \geqslant n(1-\ln 2) .
$$

The last inequality constituted a very powerful result which implies in particular the well known Heisenberg-Pauli-Weyl uncertainly principle [17].

In this paper, we consider the singular differential operator defined on $] 0,+\infty[$ by

$$
\ell_{\alpha}=\frac{\mathrm{d}^{2}}{\mathrm{dr}^{2}}+\frac{2 \alpha+1}{\mathrm{r}} \frac{\mathrm{d}}{\mathrm{dr}}=\frac{1}{\mathrm{r}^{2 \alpha+1}} \frac{\mathrm{d}}{\mathrm{dr}}\left[\mathrm{r}^{2 \alpha+1} \frac{\mathrm{d}}{\mathrm{dr}}\right] ; \alpha \geqslant \frac{-1}{2} .
$$

The Hankel transform associated with the operator $\ell_{\alpha}$ is defined by

$$
\mathscr{H}_{\alpha}(f)(\lambda)=\int_{0}^{+\infty} f(r) j_{\alpha}(\lambda r) d \mu_{\alpha}(r)
$$

where

- $d \mu_{\alpha}(r)$ is the measure defined on $[0,+\infty[$ by

$$
d \mu_{\alpha}(r)=\frac{r^{2 \alpha+1} d r}{2^{\alpha} \Gamma(\alpha+1)}
$$

- $j_{\alpha}$ is the modified Bessel-function that will be defined in the second section .

Many harmonic analysis results have been establish for the Hankel transform $\mathscr{H}_{\alpha}[14,19,20]$.

Also, many uncertainly principles have been proved for the transform $\mathscr{H}_{\alpha}[17,21]$. 
Our investigation in this work consists to establish the uncertainly principle in terms of entropy for the Hankel transform $\mathscr{H}_{\alpha}$.

For a nonnegative measurable function $f$ on $[0,+\infty[$, the entropy of $f$ is defined by

$$
E_{\mu_{\alpha}}(f)=-\int_{0}^{+\infty} f(r) \ln (f(r)) d \mu_{\alpha}(r) .
$$

Then using the Hausdorff-Young inequality for $\mathscr{H}_{\alpha}[9]$, we establish the main result of this work.

- Let $\mathrm{f} \in \mathrm{L}^{2}\left(\mathrm{~d} \mu_{\alpha}\right) ;\|\mathrm{f}\|_{2, \mu_{\alpha}}=1$ such that

$$
\int_{0}^{+\infty}|f(r)|^{2}|\ln (|f(r)|)| d \mu_{\alpha}(r)<+\infty
$$

and

$$
\int_{0}^{+\infty}\left|\mathscr{H}_{\alpha}(f)(\lambda)\right|^{2}\left|\ln \left(\left|\mathscr{H}_{\alpha}(f)(\lambda)\right|\right)\right| \mathrm{d} \mu_{\alpha}(\lambda)<+\infty .
$$

Then

$$
E_{\mu_{\alpha}}\left(|f|^{2}\right)+E_{\mu_{\alpha}}\left(\left|\mathscr{H}_{\alpha}(f)\right|^{2}\right) \geqslant(2 \alpha+1)(1-\ln 2),
$$

where $\mathrm{L}^{\mathrm{p}}\left(\mathrm{d} \mu_{\alpha}\right) ; p \in[1,+\infty]$, is the Lebesgue space of measurable functions on $[0,+\infty[$ such that

$$
\|\mathbf{f}\|_{p, \mu_{\alpha}}<+\infty
$$

with

$$
\|\mathbf{f}\|_{p, \mu_{\alpha}}= \begin{cases}\left(\int_{0}^{+\infty}|\mathrm{f}(\mathrm{r})|^{p} \mathrm{~d} \mu_{\alpha}(\mathrm{r})\right)^{\frac{1}{\mathfrak{p}}}, & \text { if } \mathrm{p} \in[1,+\infty[, \\ \underset{r \in[0,+\infty[}{\operatorname{ess} \sup _{\mathrm{r}}|\mathrm{f}(\mathrm{r})|,} & \text { if } \mathrm{p}=+\infty .\end{cases}
$$

Using this result, we prove that we can derive the Heisenberg -Pauli-Weyl inequality for $\mathscr{H}_{\alpha}$, that is

. For every $f \in L^{2}\left(d \mu_{\alpha}\right)$; we have

$$
\|\mathrm{rf}\|_{2, \mu_{\alpha}}\left\|\lambda \mathscr{H}_{\alpha}(\mathrm{f})\right\|_{2, \mu_{\alpha}} \geqslant(\alpha+1)\|f\|_{2, \mu_{\alpha}}^{2} .
$$

\section{The Hankel operator}

In this section, we recall some harmonic analysis results related to the convolution product and the Fourier transform associated with Hankel operator.

Let $\ell_{\alpha}$ be the Bessel operator defined on $] 0+\infty[$ by

$$
\ell_{\alpha} u=\frac{d^{2}}{d r^{2}} u+\frac{2 \alpha+1}{r} \frac{d u}{d r}
$$


Then, for every $\lambda \in \mathbb{C}$, the following system

$$
\left\{\begin{array}{l}
\ell_{\alpha} u(x)=-\lambda^{2} u(x) \\
u(0)=1 \\
u^{\prime}(0)=0
\end{array}\right.
$$

admits a unique solution given by $j_{\alpha}(\lambda$.$) , where$

$$
\begin{aligned}
j_{\alpha}(z) & =\frac{2^{\alpha} \Gamma(\alpha+1)}{z^{\alpha}} J_{\alpha}(z) \\
& =\Gamma(\alpha+1) \sum_{k=0}^{+\infty} \frac{(-1)^{k}}{k ! \Gamma(\alpha+k+1)}\left(\frac{z}{2}\right)^{2 k}
\end{aligned}
$$

with $J_{\alpha}$ is the Bessel function of first kind and index $\alpha[7,8,15,22]$.

The modified Bessel function $j_{\alpha}$ satisfies the following properties

for every $\alpha \geqslant-\frac{1}{2}$, the modified Bessel function $j_{\alpha}$ has the Mehler integral representation, for every $z \in \mathbb{C}$,

$$
j_{\alpha}(z)=\left\{\begin{array}{l}
\frac{2 \Gamma(\alpha+1)}{\sqrt{\pi} \Gamma\left(\alpha+\frac{1}{2}\right)} \int_{0}^{1}\left(1-t^{2}\right)^{\alpha-\frac{1}{2}} \cos z t d t, \text { if } \alpha>\frac{-1}{2} \\
\cos z, \text { if } \alpha=\frac{-1}{2} .
\end{array}\right.
$$

Consequently, for every $k \in \mathbb{N}$ and $z \in \mathbb{C}$; we have

$$
\left|\mathfrak{j}_{\alpha}^{(k)}(z)\right| \leqslant \exp (|\operatorname{Im} z|)
$$

The eigenfunction $j_{\alpha}(\lambda$.$) satisfies the following product formula [22],$ for all $r, s \in[0,+\infty[$

$$
j_{\alpha}(\lambda r) j_{\alpha}(\lambda s)=\left\{\begin{array}{l}
\frac{\Gamma(\alpha+1)}{\sqrt{\pi} \Gamma\left(\alpha+\frac{1}{2}\right)} \int_{0}^{\pi} j_{\alpha}\left(\lambda \sqrt{r^{2}+s^{2}+2 r s \cos \theta}\right) \sin ^{2 \alpha} \theta d \theta ; \text { if } \alpha>\frac{-1}{2} \\
\frac{j_{\alpha}(\lambda(r+s))+j_{\alpha}(\lambda((r-s)))}{2}, \text { if } \alpha=\frac{-1}{2} .
\end{array}\right.
$$

The previous product formula allows us to define the Hankel translation operator and the convolution product related to the operator $\ell_{\alpha}$ as follows

Definition 2.1. i) For every $r \in\left[0,+\infty\right.$, the Hankel translation operator $\tau_{r}^{\alpha}$ is defined on $\mathrm{L}^{\mathrm{p}}\left(\mathrm{d} \mu_{\alpha}\right) ; p \in[1,+\infty]$, by

$$
\begin{aligned}
& \tau_{r}^{\alpha} f(s)= \\
& \left\{\begin{array}{l}
\frac{\Gamma(\alpha+1)}{\sqrt{\pi} \Gamma\left(\alpha+\frac{1}{2}\right)} \int_{0}^{\pi} f\left(\sqrt{r^{2}+s^{2}+2 r \cos \theta}\right) \sin ^{2 \alpha} \theta d \theta ; \text { if } \alpha>\frac{-1}{2} \\
\frac{f(r+s)+f(|r-s|)}{2}, \text { if } \alpha=\frac{-1}{2} .
\end{array}\right.
\end{aligned}
$$


ii) The convolution product of $f, g \in L^{1}\left(d \mu_{\alpha}\right)$ is defined for every $r \in[0,+\infty[$, by

$$
f *_{\alpha} g(r)=\int_{0}^{+\infty} \tau_{r}^{\alpha}(f)(s) g(s) d \mu_{\alpha}(s) .
$$

Then the product formula (2.3) can be written

$$
\tau_{r}^{\alpha}\left(j_{\alpha}(\lambda .)\right)(s)=j_{\alpha}(\lambda r) j_{\alpha}(\lambda s) .
$$

We have the properties

Proposition 2.2. i. For every $f \in \mathrm{L}^{\mathrm{p}}\left(\mathrm{d} \mu_{\alpha}\right) ; 1 \leqslant p \leqslant+\infty$, and for every $\mathrm{r} \in[0,+\infty$, the function $\tau_{r}^{\alpha}(f)$ belongs to $\mathrm{L}^{\mathfrak{p}}\left(\mathrm{d} \mu_{\alpha}\right)$ and we have

$$
\left\|\tau_{r}^{\alpha}(f)\right\|_{p, \mu_{\alpha}} \leqslant\|f\|_{p, \mu_{\alpha}} .
$$

ii. For $\mathrm{f}, \mathrm{g} \in \mathrm{L}^{1}\left(\mathrm{~d} \mu_{\alpha}\right)$, the function $\mathrm{f} *_{\alpha} \mathrm{g}$ belongs to $\mathrm{L}^{1}\left(\mathrm{~d} \mu_{\alpha}\right)$; the convolution product is commutative, associative and we have

$$
\left\|\mathbf{f} *_{\alpha} g\right\|_{1, \mu_{\alpha}} \leqslant\|f\|_{1, \mu_{\alpha}}\|g\|_{1, \mu_{\alpha}} .
$$

Moreover, if $1 \leqslant \mathrm{p}, \mathrm{q}, \mathrm{r} \leqslant+\infty$ are such that $\frac{1}{\mathrm{r}}=\frac{1}{\mathrm{p}}+\frac{1}{\mathrm{q}}-1$ and if

$f \in L^{p}\left(d \mu_{\alpha}\right), g \in L^{q}\left(d \mu_{\alpha}\right)$, then the function $f *_{\alpha} g$ belongs to $L^{r}\left(d \mu_{\alpha}\right)$, and we have the Young's inequality

$$
\left\|f *_{\alpha} g\right\|_{r, \mu_{\alpha}} \leqslant\|f\|_{p, \mu_{\alpha}}\|g\|_{q, \mu_{\alpha}} .
$$

iii. For every $\mathrm{f} \in \mathrm{L}^{1}\left(\mathrm{~d} \mu_{\alpha}\right)$, and $\mathrm{r} \in\left[0,+\infty\left[\right.\right.$ the function $\tau_{r}^{\alpha}(\mathrm{f})$ belongs to $\mathrm{L}^{1}\left(\mathrm{~d} \mu_{\alpha}\right)$ and we have

$$
\int_{0}^{+\infty} \tau_{r}^{\alpha}(f)(s) d \mu_{\alpha}(s)=\int_{0}^{+\infty} f(r) d \mu_{\alpha}(r) .
$$

We denoted by

- $\mathscr{C}_{*, 0}(\mathbb{R})$ the space of even continuous functions $f$ on $\mathbb{R}$ such that

$$
\lim _{|r| \rightarrow+\infty} f(r)=0 .
$$

- $\mathscr{S}_{\mathrm{e}}(\mathbb{R})$ the space of even infinitely differentiable functions on $\mathbb{R}$; rapidly decreasing together with all their derivatives.

Now, we shall define the Hankel transform and we give the most important properties.

Definition 2.3. The Hankel transform $\mathscr{H}_{\alpha}$ is defined on $\mathrm{L}^{1}\left(\mathrm{~d} \mu_{\alpha}\right)$ by

$$
\forall \lambda \in \mathbb{R} ; \mathscr{H}_{\alpha}(f)(\lambda)=\int_{0}^{+\infty} f(r) j_{\alpha}(\lambda r) d \mu_{\alpha}(r)
$$

where $j_{\alpha}$ is the modified Bessel function defined by the relation(2.1). 
Proposition 2.4. $\quad$ i. For every $\mathrm{f} \in \mathrm{L}^{1}\left(\mathrm{~d} \mu_{\alpha}\right)$, the function $\mathscr{H}_{\alpha}(\mathrm{f})$ belongs to the space $\mathscr{C}_{*, 0}(\mathbb{R})$ and

$$
\left\|\mathscr{H}_{\alpha}(f)\right\|_{\infty, \mu_{\alpha}} \leqslant\|f\|_{1, \mu_{\alpha}} .
$$

ii. For every $\mathrm{f} \in \mathrm{L}^{1}\left(\mathrm{~d} \mu_{\alpha}\right)$ and $\mathrm{r} \in[0,+\infty[$,

$$
\mathscr{H}_{\alpha}\left(\tau_{r}^{\alpha}(f)\right)(\lambda)=j_{\alpha}(\lambda r) \mathscr{H}_{\alpha}(f)(\lambda) .
$$

iii. For all $\mathrm{f}, \mathrm{g} \in \mathrm{L}^{1}\left(\mathrm{~d} \mu_{\alpha}\right)$,

$$
\mathscr{H}_{\alpha}\left(\mathrm{f} *_{\alpha} \mathrm{g}\right)(\lambda)=\mathscr{H}_{\alpha}(\mathrm{f})(\lambda) \mathscr{H}_{\alpha}(\mathrm{g})(\lambda) .
$$

Theorem 2.5. (Inversion formula) Let $\mathrm{f} \in \mathrm{L}^{1}\left(\mathrm{~d} \mu_{\alpha}\right)$ such that $\mathscr{H}_{\alpha}(\mathrm{f}) \in \mathrm{L}^{1}\left(\mathrm{~d} \mu_{\alpha}\right)$, then for almost every $r \in[0,+\infty[$, we have

$$
f(r)=\int_{0}^{+\infty} \mathscr{H}_{\alpha}(f)(\lambda) j_{\alpha}(\lambda r) \mathrm{d} \mu_{\alpha}(\lambda)=\mathscr{H}_{\alpha}\left(\mathscr{H}_{\alpha}(\mathrm{f})\right)(\mathrm{r}) .
$$

Theorem 2.6. (Plancherel) The Hankel transform $\mathscr{H}_{\alpha}$ can be extented to an isometric isomorphism from $\mathrm{L}^{2}\left(\mathrm{~d} \mu_{\alpha}\right)$ onto itself. In particular, for all $\mathrm{f}$ and $\mathrm{g} \in \mathrm{L}^{2}\left(\mathrm{~d} \mu_{\alpha}\right)$, we have (Parseval equality)

$$
\int_{0}^{+\infty} \mathrm{f}(\mathrm{r}) \overline{\mathrm{g}(\mathrm{r})} \mathrm{d} \mu_{\alpha}(\mathrm{r})=\int_{0}^{+\infty} \mathscr{H}_{\alpha}(\mathrm{f})(\lambda) \overline{\mathscr{H}_{\alpha}(\mathrm{g})(\lambda)} \mathrm{d} \mu_{\alpha}(\lambda) .
$$

Proposition 2.7. i. Let $\mathrm{f} \in \mathrm{L}^{1}\left(\mathrm{~d} \mu_{\alpha}\right)$ and $\mathrm{g} \in \mathrm{L}^{2}\left(\mathrm{~d} \mu_{\alpha}\right)$; by the relation (2.8), the function $\mathrm{f} *_{\alpha} \mathrm{g}$ belongs to $\mathrm{L}^{2}\left(\mathrm{~d} \mu_{\alpha}\right)$, moreover

$$
\mathscr{H}_{\alpha}\left(\mathrm{f} *_{\alpha} \mathrm{g}\right)(\lambda)=\mathscr{H}_{\alpha}(\mathrm{f})(\lambda) \mathscr{H}_{\alpha}(\mathrm{g})(\lambda) .
$$

ii. For all $\mathrm{f}$ and $\mathrm{g} \in \mathrm{L}^{2}\left(\mathrm{~d} \mu_{\alpha}\right)$, the function $\mathrm{f} *_{\alpha} \mathrm{g}$ belongs to $\mathscr{C}_{*, 0}(\mathbb{R})$ and we have

$$
\mathrm{f} *_{\alpha} \mathrm{g}=\mathscr{H}_{\alpha}\left(\mathscr{H}_{\alpha}(\mathrm{f}) \cdot \mathscr{H}_{\alpha}(\mathrm{g})\right) \text {. }
$$

iii. The Hankel transform $\mathscr{H}_{\alpha}$ is a topological isomorphism from $\mathscr{S}_{\mathrm{e}}(\mathbb{R})$ onto itself and we have

$$
\mathscr{H}_{\alpha}^{-1}=\mathscr{H}_{\alpha}
$$

iv. For every $\mathrm{f} \in \mathscr{S}(\mathbb{R})$ and $\mathrm{g} \in \mathrm{L}^{2}\left(\mathrm{~d} \mu_{\alpha}\right)$, we have

$$
\mathscr{H}_{\alpha}(\mathrm{fg})(\lambda)=\mathscr{H}_{\alpha}(\mathrm{f})(\lambda) *_{\alpha} \mathscr{H}_{\alpha}(\mathrm{g})(\lambda) .
$$

Definition 2.8. The Gaussian kernel associated with the Hankel operator is defined by

$$
\forall t>0, g_{t}(r)=\frac{e^{\frac{-r^{2}}{2 t^{2}}}}{t^{2 \alpha+2}} .
$$

Thus, one can easily see that the family $\left(g_{t}\right)_{t>0}$ is an approximation of the identity, in particular, for every $f \in L^{2}\left(d \mu_{\alpha}\right)$ we have

$$
\lim _{t \rightarrow 0^{+}}\left\|g_{t} *_{\alpha} f-f\right\|_{2, \mu_{\alpha}}=0 .
$$




\section{Uncertainty principle in terms of entropy for the Hankel transform}

This section contains the main result of this paper that is the uncertainty principle in terms of entropy for the Hankel transform $\mathscr{H}_{\alpha}$. We start this section by the following Hausdorff-Young inequality.

Theorem 3.1. [9] Let $\mathrm{p} \in] 1,2]$, for every $\mathrm{f} \in \mathrm{L}^{\mathrm{p}}\left(\mathrm{d} \mu_{\alpha}\right)$, the function $\mathscr{H}_{\alpha}(\mathrm{f})$ belongs to $\mathrm{L}^{\mathrm{p}^{\prime}}\left(\mathrm{d} \mu_{\alpha}\right) ; \mathrm{p}^{\prime}=$ $\frac{\mathrm{p}}{\mathrm{p}-1}$, and we have

$$
\left\|\mathscr{H}_{\alpha}(f)\right\|_{p^{\prime}, \mu_{\alpha}} \leqslant A_{p, \alpha}\|f\|_{p, \mu_{\alpha}},
$$

where $A_{p, \alpha}$ is the Babenko-Beckner constant,

$$
A_{p, \alpha}=\left(\frac{p^{\frac{1}{p}}}{\left(\frac{p}{p-1}\right)^{\frac{p-1}{p}}}\right)^{\alpha+1} .
$$

Lemma 3.2. Let $x$ be a positive real number. For every $\mathrm{p} \in[1,2[$,

$$
x^{2}-x \leqslant \frac{x^{p}-x^{2}}{p-2} \leqslant x^{2} \ln x
$$

Proof. Let $x>0$ and let us put

$$
g(p)=\frac{x^{p}-x^{2}}{p-2}
$$

$g$ is differentiable on $\left[1,2\left[\right.\right.$ and we have $g^{\prime}(p)=\frac{h(p)}{(p-2)^{2}}$,

where $h(p)=(p-2) \ln (x) \cdot x^{p}-x^{p}+x^{2}$.

On the other hand,

$$
\forall p \in\left[1,2\left[; h^{\prime}(p)=(p-2) x^{p}(\ln (x))^{2}<0\right.\right.
$$

and $h(2)=0$.

Thus, for every $p \in[1,2], h(p) \geqslant 0$ and the function $g$ is increasing on $[1,2[$, hence

$$
g(1) \leqslant g(p) \leqslant \lim _{p \rightarrow 2^{-}} g(p)
$$

In the following, we shall prove the uncertainty principle in terms of entropy for $f \in L^{1}\left(d \mu_{\alpha}\right) \cap$ $\mathrm{L}^{2}\left(\mathrm{~d} \mu_{\alpha}\right)$ such that $\|\mathrm{f}\|_{2, \mu_{\alpha}}=1$. 
Theorem 3.3. Let $\mathrm{f} \in \mathrm{L}^{1}\left(\mathrm{~d} \mu_{\alpha}\right) \cap \mathrm{L}^{2}\left(\mathrm{~d} \mu_{\alpha}\right) ;\|\mathrm{f}\|_{2, \mu_{\alpha}}=1$, such that

$$
\int_{0}^{\infty}|f(r)|^{2}|\ln (|f(r)|)| d \mu_{\alpha}(r)<+\infty
$$

and

$$
\int_{0}^{\infty}|\mathscr{H}(\boldsymbol{f})(\lambda)|^{2}|\ln (|\mathscr{H}(\boldsymbol{f})(\lambda)|)| \mathrm{d} \mu_{\alpha}(\lambda)<+\infty
$$

Then,

$$
E_{\mu_{\alpha}}\left(|f|^{2}\right)+E_{\mu_{\alpha}}\left(\left|\mathscr{H}_{\alpha}(f)\right|^{2}\right) \geqslant(2 \alpha+2)(1-\ln 2) .
$$

Proof. Let $\mathrm{f} \in \mathrm{L}^{1}\left(\mathrm{~d} \mu_{\alpha}\right) \cap \mathrm{L}^{2}\left(\mathrm{~d} \mu_{\alpha}\right) ;\|f\|_{2, \mu_{\alpha}}=1$ and let $\varphi$ be the function defined on $\left.] 1,2\right]$ by

$$
\varphi(p)=\int_{0}^{+\infty}\left|\mathscr{H}_{\alpha}(f)(\lambda)\right|^{\frac{p}{p-1}} d \mu_{\alpha}(\lambda)-\left(\frac{\frac{1}{p}^{\frac{1}{p}}}{\left(\frac{p}{p-1}\right)^{\frac{p-1}{p}}}\right)^{\frac{p(\alpha+1)}{p-1}}\left(\int_{0}^{+\infty}|f(x)|^{p} d \mu_{\alpha}(x)\right)^{\frac{1}{p-1}}
$$

Then, by relation (3.1),

$$
\forall p \in] 1,2] ; \varphi(p) \leqslant 0 .
$$

On the other hand, Theorem 2.6 means that $\varphi(2)=0$. This implies that

$$
\frac{\mathrm{d} \varphi}{\mathrm{dp}}\left(2^{-}\right) \geqslant 0
$$

Since $f \in L^{1}\left(d \mu_{\alpha}\right) \cap L^{2}\left(d \mu_{\alpha}\right)$, then by a standard interpolation argument, $f$ belongs to $L^{p}\left(d \mu_{\alpha}\right) ; p \in$ $[1,2]$.

Using Lemma 3.2, the hypothesis and Lebesgue dominated convergence theorem, we deduce that

$$
\frac{d}{d p}\left[\int_{0}^{+\infty}|f(r)|^{p} d \mu_{\alpha}(r)\right]\left(2^{-}\right)=\int_{0}^{+\infty} \lim _{p \rightarrow 2^{-}} \frac{|f(r)|^{p}-|f(r)|^{2}}{p-2} d \mu_{\alpha}(r) .
$$

Thus

$$
\left.\frac{\mathrm{d}}{\mathrm{dp}}\left[\int_{0}^{+\infty}|\mathrm{f}(\mathrm{r})|^{p} \mathrm{~d} \mu_{\alpha}(\mathrm{r})\right]\left(2^{-}\right)=\frac{1}{2} \int_{0}^{+\infty} \ln |\mathrm{f}(\mathrm{r})|^{2}|\mathrm{f}(\mathrm{r})|^{2} \mathrm{~d} \mu_{\alpha}(\mathrm{r})\right]
$$

Now, since $f \in L^{1}\left(d \mu_{\alpha}\right) \cap L^{2}\left(d \mu_{\alpha}\right)$, by using again the Lebesgue dominated convergence theorem, we get

$$
\lim _{p \rightarrow 2} \int_{0}^{+\infty}|f(r)|^{p} d \mu_{\alpha}(r)=\int_{0}^{+\infty}|f(r)|^{2} d \mu_{\alpha}(r)=1
$$

Combining (3.6) and (3.7), we get

$$
\frac{d}{d p}\left[\left(\int_{0}^{+\infty}|f(r)|^{p} d \mu_{\alpha}(r)\right)^{\frac{1}{p-1}}\right]\left(2^{-}\right)=-\frac{1}{2} E_{\mu_{\alpha}}\left(|f|^{2}\right) .
$$


As the same way, one can see that

$$
\frac{\mathrm{d}}{\mathrm{dp}}\left[\int_{0}^{+\infty}\left|\mathscr{H}_{\alpha}(f)(\lambda)\right|^{\frac{\mathrm{p}}{\mathrm{p}-1}} \mathrm{~d} \mu_{\alpha}(\lambda)\right]\left(2^{-}\right)=-\frac{1}{2} \mathrm{E}_{\mu_{\alpha}}\left(\left|\mathscr{H}_{\alpha}(f)\right|^{2}\right) .
$$

Finally, basic calculations show that

$$
\frac{d}{d p}\left[\left(\frac{\frac{1}{p}}{\left(\frac{p}{p-1}\right)^{\frac{p-1}{p}}}\right)^{\frac{p}{p-1}}(\alpha+1)\right]\left(2^{-}\right)=(\alpha+1)(1-\ln 2) .
$$

Thus according to relations(3.8), (3.9) and (3.10), it follows that

$$
\frac{\mathrm{d} \varphi}{\mathrm{dp}}\left(2^{-}\right)=\frac{1}{2} \mathrm{E}_{\mu_{\alpha}}\left(|\mathrm{f}|^{2}\right)+\frac{1}{2} \mathrm{E}_{\mu_{\alpha}}\left(\left|\mathscr{H}_{\alpha}(\mathrm{f})\right|^{2}\right)-(\alpha+1)(1-\ln 2) .
$$

The proof is complete by using (3.4).

Lemma 3.4. Let $\mathrm{f}$ be a measurable function on $[0,+\infty[$ and let

$$
\omega:[0,+\infty[\longmapsto[0,+\infty[
$$

be a convex function such that $\boldsymbol{\omega}(|\mathbf{f}|)$ belongs to $\mathrm{L}^{1}\left(\mathrm{~d} \boldsymbol{\mu}_{\alpha}\right)$.

Let $\left(\mathrm{f}_{\mathrm{k}}\right)_{\mathrm{k} \in \mathbb{N}}$ be a sequence of nonnegative measurable functions on $\mathbb{R}_{+}$such that for every $\mathrm{k} \in \mathbb{N}$; $\left\|f_{k}\right\|_{1, \mu_{\alpha}}=1$ and the sequence $\left(f_{k} *_{\alpha} f\right)_{k \in \mathbb{N}}$ converges pointwise to $f$.

Then, for every $k \in \mathbb{N}$, the function $\omega\left(\left|f_{k} *_{\alpha} f\right|\right.$ belongs to $L^{1}\left(d \mu_{\alpha}\right)$, and we have

$$
\lim _{k \rightarrow+\infty} \int_{0}^{+\infty} \omega\left(\left|f_{k} *_{\alpha} f\right|\right)(r) d \mu_{\alpha}(r)=\int_{0}^{+\infty} \omega(|f(r)|) d \mu_{\alpha}(r) .
$$

Proof. We have

$$
\|\omega \circ|f|\|_{1, \mu_{\alpha}}=\int_{0}^{+\infty} \liminf _{k \rightarrow+\infty} \omega\left(\left|f_{k} *_{\alpha} f\right|\right)(r) d \mu_{\alpha}(r)
$$

and by using Fatou's lemma, we get

$$
\|\omega \circ|f|\|_{1, \alpha} \leqslant \liminf _{p \rightarrow+\infty} \int_{o}^{+\infty} \omega\left(\left|f_{k} *_{\alpha} f(r)\right|\right) d \mu_{\alpha}(r) .
$$

Conversely, according to relation (2.9), we have

$$
\forall \mathrm{k} \in \mathbb{N} ; \forall \lambda \in \mathbb{R}_{+}, \int_{0}^{+\infty} \tau_{\lambda}^{\alpha}\left(f_{k}\right)(r) d \mu_{\alpha}(r)=\left\|f_{k}\right\|_{1, \mu_{\alpha}}=1,
$$

which means that for every $\lambda \in \mathbb{R}_{+}, \tau_{\lambda}^{\alpha}\left(f_{k}\right)(r) d \mu_{\alpha}(r)$ is a probability measure on $\mathbb{R}_{+}$. Therefore, by using Jensen's inequality for convex functions, we get 


$$
\begin{aligned}
\forall r \in \mathbb{R}_{+}, \omega\left(\left|f_{k} *_{\alpha} f(r)\right|\right) & =\omega\left(\mid \int_{0}^{+\infty} f(x) \tau_{r}^{\alpha}\left(f_{k}\right)(x) d \mu_{\alpha}(x)\right) \\
& \leqslant \omega\left(\int_{0}^{+\infty}\left|f(x) \tau_{r}^{\alpha}\left(f_{k}\right)(x)\right| d \mu_{\alpha}(x)\right. \\
& \leqslant \int_{0}^{+\infty} \omega(|f(x)|) \tau_{r}^{\alpha}\left(f_{k}\right)(x) d \mu_{\alpha}(x) \\
& =f_{k} *_{\alpha}(\omega \circ|f|)(r) .
\end{aligned}
$$

In particular, $\omega \circ\left|f_{k} *_{\alpha} f\right| \in L^{1}\left(d \mu_{\alpha}\right)$. Hence by relations (2.9) and (3.16), we deduce that

$$
\begin{aligned}
\limsup _{k \rightarrow+\infty} \int_{0}^{+\infty} \omega\left(\left|f_{k} *_{\alpha} f(r)\right|\right) d \mu_{\alpha}(r) & \leqslant \limsup _{k \rightarrow+\infty} \int_{0}^{+\infty} f_{k} *_{\alpha}(\omega \circ|f(r)|) d \mu_{\alpha}(r) \\
& =\limsup _{k \rightarrow+\infty}\left\|f_{k} *_{\alpha}(\omega \circ|f|)\right\|_{1, \alpha} \\
& \leqslant\|\omega \circ|f|\|_{1, \alpha .}
\end{aligned}
$$

The relations (3.14) and (3.17) show that

$$
\lim _{k \rightarrow+\infty} \int_{0}^{+\infty} \omega\left(\left|f_{k} *_{\alpha} f\right|\right)(r) d \mu_{\alpha}(r)=\int_{0}^{+\infty} \omega(|f(r)|) d \mu_{\alpha}(r) .
$$

Now, e are able to prove the main result.

Theorem 3.5. Let $\mathrm{f} \in \mathrm{L}^{2}\left(\mathrm{~d} \mu_{\alpha}\right) ;\|\mathrm{f}\|_{2, \mu_{\alpha}}=1$, such that

$$
\int_{0}^{\infty}|f(r)|^{2}|\ln (|f(r)|)| d \mu_{\alpha}(r)<+\infty
$$

and

$$
\int_{0}^{\infty}\left|\mathscr{H}_{\alpha}(\mathrm{f})(\lambda)\right|^{2}\left|\ln \left(\left|\mathscr{H}_{\alpha}(\mathrm{f})(\lambda)\right|\right)\right| \mathrm{d} \mu_{\alpha}(\lambda)<+\infty
$$

Then, we have

$$
E_{\mu_{\alpha}}\left(|f|^{2}\right)+E_{\mu_{\alpha}}\left(\left|\mathscr{H}_{\alpha}(f)\right|^{2}\right) \geqslant(2 \alpha+2)(1-\ln 2) .
$$

Proof. The main idea of this proof is to combine Theorem 3.3 with the standard density argument. Indeed, we will show that for every $f \in \mathrm{L}^{2}\left(\mathrm{~d} \mu_{\alpha}\right)$; there exists a sequence $\left(f_{k}\right)_{k \in \mathbb{N}} \in L^{1}\left(d \mu_{\alpha}\right) \cap L^{2}\left(d \mu_{\alpha}\right)$ such that

$$
\lim _{k \rightarrow+\infty}\left\|f_{k}\right\|_{2, \mu_{\alpha}}=\|f\|_{2, \mu_{\alpha}}
$$




$$
\begin{aligned}
\lim _{k \rightarrow+\infty} E_{\mu_{\alpha}}\left(\left|f_{k}\right|^{2}\right) & =E_{\mu_{\alpha}}\left(|f|^{2}\right), \\
\lim _{k \rightarrow+\infty} E_{\alpha}\left(\left|\mathscr{H}_{\alpha}\left(f_{k}\right)\right|^{2}\right) & =E_{\alpha}\left(\left|\mathscr{H}_{\alpha}(f)\right|^{2}\right) .
\end{aligned}
$$

Let $\left(h_{k}\right)_{k \in \mathbb{N}}$ be the sequence of functions defined by

$$
h_{k}(r)=2^{\alpha+1} k^{2 \alpha+2} e^{-k^{2} r^{2}}=g_{\frac{1}{k \sqrt{2}}}(r) .
$$

Then, by relation(2.21), we have

$$
\forall \mathrm{f} \in \mathrm{L}^{2}\left(\mathrm{~d} \mu_{\alpha}\right) ; \lim _{k \rightarrow+\infty}\left\|\mathrm{h}_{\mathrm{k}} *_{\alpha} \mathrm{f}-\mathrm{f}\right\|_{2, \mu_{\alpha}}=0 .
$$

Furthermore, according to Weber's formula [15], we know that for all $k \in \mathbb{N}^{*}, \alpha \geqslant \frac{-1}{2}$,

$$
\int_{0}^{+\infty} e^{-k^{2} r^{2}} j_{\alpha}(x r) r^{2 \alpha+1} d r=\Gamma(\alpha+1) \frac{e^{-\frac{x^{2}}{4 k^{2}}}}{2 k^{2 \alpha+2}} .
$$

Hence, by relation (3.24), we deduce that

$$
\begin{aligned}
\mathscr{H}_{\alpha}^{-1}\left(h_{k}\right)(\lambda) & =\frac{2 k^{2 \alpha+2}}{\Gamma(\alpha+1)} \int_{0}^{+\infty} e^{-k^{2} r^{2}} j_{\alpha}(\lambda r) r^{2 \alpha+1} d \mu_{\alpha}(r) \\
& =e^{-\frac{\lambda^{2}}{4 k^{2}}}
\end{aligned}
$$

Let $\left(\psi_{k}\right)_{k \in \mathbb{N}}$ be the sequence of functions defined on $\mathbb{R}_{+}$by

$$
\psi_{k}(\lambda)=e^{-\frac{\lambda^{2}}{4 k^{2}}}=\mathscr{H}_{\alpha}^{-1}\left(h_{k}\right)(\lambda)
$$

Let $f \in \mathrm{L}^{2}\left(\mathrm{~d} \mu_{\alpha}\right) ;\|f\|_{2, \mu_{\alpha}}=1$, then according to relation(3.23), we have

$$
\lim _{k \rightarrow+\infty}\left\|\mathscr{H}_{\alpha}\left(\psi_{k}\right) *_{\alpha} \mathscr{H}_{\alpha}(\mathrm{f})-\mathscr{H}_{\alpha}(\mathrm{f})\right\|_{2, \mu_{\alpha}}=0 .
$$

In particular, there is a subsequence $\left(\psi_{\sigma(k)}\right)_{k \in \mathbb{N}}$ such that $\mathscr{H}_{\alpha}\left(\psi_{\sigma(\mathrm{k})}\right) *_{\alpha} \mathscr{H}_{\alpha}(\mathrm{f})=\mathrm{h}_{\sigma(\mathrm{k})} *_{\alpha} \mathscr{H}_{\alpha}(\mathrm{f})$ converges pointwise to $\mathscr{H}_{\alpha}(\mathrm{f})$.

Let $\left(f_{k}\right)_{k \in \mathbb{N}}$ be the sequence of measurable functions on $\mathbb{R}_{+}$defined by

$$
f_{k}=\psi_{\sigma(k)} f .
$$

Since $\psi_{\sigma(k)} \in L^{2}\left(d \mu_{\alpha}\right) \cap C_{*, 0}\left(\mathbb{R}_{+}\right)$, then $f_{k}$ belongs to the space $L^{1}\left(d \mu_{\alpha}\right) \cap L^{2}\left(d \mu_{\alpha}\right)$. Replacing $f$ by $\frac{f_{k}}{\left\|f_{k}\right\|_{2, \mu_{\alpha}}}$ in Theorem 3.3 and using the fact that

$$
\|f\|_{2, \mu_{\alpha}}=\left\|\mathscr{H}_{\alpha}(\mathrm{f})\right\|_{2, \mu_{\alpha}} ; f \in \mathrm{L}^{2}\left(\mathrm{~d} \mu_{\alpha}\right),
$$


we deduce that

$$
\begin{array}{r}
-\int_{0}^{+\infty} \ln \left(\left|f_{k}(r)\right|^{2}\right)\left|f_{k}(r)\right|^{2} \mathrm{~d} \mu_{\alpha}(r)-\int_{0}^{+\infty} \ln \left(\left|\mathscr{H}_{\alpha}\left(f_{k}\right)(\lambda)\right|^{2}\right)\left|\mathscr{H}_{\alpha}\left(f_{k}\right)(\lambda)\right|^{2} \mathrm{~d} \mu_{\alpha}(\lambda) \\
\geqslant(2 \alpha+2)(1-\ln 2)\left\|f_{k}\right\|_{2, \mu_{\alpha}}^{2}-2\left\|f_{k}\right\|_{2, \alpha}^{2} \ln \left(\left\|f_{k}\right\|_{2, \mu_{\alpha}}^{2}\right) .
\end{array}
$$

Now, by using the Lebesgue dominated convergence theorem, we have

$$
\lim _{k \rightarrow+\infty}\left\|f_{k}\right\|_{2, \mu_{\alpha}}=\|f\|_{2, \mu_{\alpha}} .
$$

On the other hand, one can see that for all $k \in \mathbb{N}$, and for almost every $r \in[0,+\infty[$, we have

$$
\ln \left|f_{k}(r)\right|^{2}\left|f_{k}(r)\right|^{2} \leqslant C|f(r)|^{2}+\ln |f(r)|^{2}|f(r)|^{2}
$$

Hence, using again the Lebesgue dominated convergence theorem, we get

$$
-\lim _{k \rightarrow+\infty} \int_{0}^{+\infty} \ln \left(\left|f_{k}(r)\right|^{2}\right)\left|f_{k}(r)\right|^{2} d \mu_{\alpha}(r)=E_{\mu_{\alpha}}\left(|f|^{2}\right) .
$$

Now, let us show that

$$
\lim _{k \rightarrow+\infty} E_{\mu_{\alpha}}\left(\left|\mathscr{H}_{\alpha}\left(f_{k}\right)\right|^{2}\right)=E_{\mu_{\alpha}}\left(\left.\mathscr{H}_{\alpha}(f)\right|^{2}\right) .
$$

For this, we denote by $\omega_{1}, \omega_{2}$ the functions defined on $\mathbb{R}$ by

$\omega_{1}(t)= \begin{cases}t^{2} \ln |t|, & \text { if }|t|>1 \\ 0, & \text { if }|t| \leqslant 1\end{cases}$

and

$\omega_{2}(t)= \begin{cases}2 t^{2}, & \text { if }|t| \geqslant 1 \\ -t^{2} \ln |t|+2 t^{2}, & \text { if }|t| \leqslant 1, t \neq 0 \\ 0, & \text { if } t=0 .\end{cases}$

Then $\omega_{1}$ and $\omega_{2}$ are both nonnegative and convex, moreover; we have

$$
\forall \mathrm{t}>0, \mathrm{t}^{2} \ln |\mathrm{t}|=\omega_{1}(\mathrm{t})-\omega_{2}(\mathrm{t})+2 \mathrm{t}^{2} .
$$

From the hypothesis, for each $i \in\{1,2\}$, the function $\omega_{i}\left(\left|\mathscr{H}_{\alpha}(f)\right|\right)$ belongs to $L^{1}\left(d \mu_{\alpha}\right)$. Now, from Proposition $2.7 \mathrm{iv}$ ), for every $k \in \mathbb{N}^{*}$; we have

$$
\mathscr{H}_{\alpha}\left(f_{k}\right)=h_{\sigma(k)} *_{\alpha} \mathscr{H}_{\alpha}(f)
$$

and we know that $h_{\sigma(k)} *_{\alpha} \mathscr{H}_{\alpha}(f)$ converges pointwise to $\mathscr{H}_{\alpha}(f)$ and $\left\|h_{\sigma(k)}\right\|_{1, \mu_{\alpha}}=1$. So, the hypothesis of Lemma 3.4 are satisfies and we get

$$
\lim _{k \rightarrow+\infty} \int_{0}^{+\infty} \omega_{i}\left(\left|\mathscr{H}_{\alpha}\left(f_{k}\right)\right|\right)(r) d \mu_{\alpha}(r)=\int_{0}^{+\infty} \omega_{i}\left(\left|\mathscr{H}_{\alpha}(f)\right|\right)(r) d \mu_{\alpha}(r),
$$

and therefore, by relations(3.30) and (3.33) we get

$$
\lim _{k \rightarrow+\infty} \int_{0}^{+\infty} \ln \left|\mathscr{H}_{\alpha}\left(f_{k}\right)\right|^{2}\left|\mathscr{H}_{\alpha}\left(f_{k}\right)(r)\right|^{2} \mathrm{~d} \mu_{\alpha}(r)=E_{\mu_{\alpha}}\left(\left|\mathscr{H}_{\alpha}(f)\right|^{2}\right) .
$$

The proof is complete by combining relations (3.29), (3.30), (3.32)and(3.35). 


\section{Heisenberg-Pauli-Weyl inequality for the Hankel tran- sorm}

Lemma 4.1. Let $\mathrm{f} \in \mathrm{L}^{2}\left(\mathrm{~d} \mu_{\alpha}\right)$ such that $\|\mathrm{f}\|_{2, \mu_{\alpha}}=1$. Then, for every $\mathrm{t}>0$,

$$
\int_{0}^{+\infty}|f(r)|^{2} \ln \left(\frac{|f(r)|^{2}}{g_{t}(r)}\right) d \mu_{\alpha}(r) \geqslant 0,
$$

where $\mathrm{g}_{\mathrm{t}}(\mathrm{r})$ is given by (2.20).

Proof. Since the function $\omega(t)=t \ln t$ is convex on $] 0,+\infty[$, and

$d v_{\alpha}(r)=g_{t}(r) d \mu_{\alpha}(r)$ is a probability measure on $] 0,+\infty[$ then, applying Jensen's inequality, we get

$$
\begin{aligned}
\int_{0}^{+\infty}|f(r)|^{2} \ln \left(\frac{|f(r)|^{2}}{g_{\mathrm{t}}(r)}\right) d \mu_{\alpha}(r) & =\int_{0}^{+\infty} \omega\left(\frac{|f(r)|^{2}}{g_{\mathfrak{t}}(r)}\right) d v_{\alpha}(r) \\
& \geqslant \omega\left(\int_{0}^{+\infty} \frac{|f(r)|^{2}}{g_{\mathfrak{t}}(r)} d v_{\alpha}(r)\right) \\
& =\omega\left(\|f\|_{2, \mu_{\alpha}}^{2}\right) \\
& =\omega(1) \\
& =0 .
\end{aligned}
$$

Theorem 4.2. (Heisenberg-Pauli-Weyl inequality)

Let $\mathrm{f} \in \mathrm{L}^{2}\left(\mathrm{~d} \mu_{\alpha}\right)$, then

$$
\|\mathrm{rf}\|_{2, \mu_{\alpha}}\left\|\lambda \mathscr{H}_{\alpha}(f)\right\|_{2, \mu_{\alpha}} \geqslant(\alpha+1)\|f\|_{2, \mu_{\alpha}}^{2}
$$

Proof. Let $\mathrm{h} \in \mathrm{L}^{2}\left(\mathrm{~d} \mu_{\alpha}\right) ;\|\mathrm{h}\|_{2, \mu_{\alpha}}=1$. From Lemma 4.1, we get

$$
\int_{0}^{+\infty}\left[|h(r)|^{2} \ln \left(|h(r)|^{2}\right)-|h(r)|^{2} \ln \left(\left|g_{t}(r)\right|\right)\right] d \mu_{\alpha}(r) \geqslant 0
$$

Then,

$$
E_{\mu_{\alpha}}\left(|h|^{2}\right) \leqslant \ln \left(t^{2 \alpha+2}\right)+\frac{1}{2 t^{2}} \int_{0}^{+\infty}|h(r)|^{2} r^{2} d \mu_{\alpha}(r) .
$$

Since $\left\|\mathscr{H}_{\alpha}(\mathrm{h})\right\|_{2, \mu_{\alpha}}=\|\mathrm{h}\|_{2, \mu_{\alpha}}=1$, we get also

$$
\mathrm{E}_{\mu_{\alpha}}\left(\left|\mathscr{H}_{\alpha}(\mathrm{h})\right|^{2}\right) \leqslant \ln \left(\mathrm{t}^{2 \alpha+2}\right)+\frac{1}{2 \mathrm{t}^{2}} \int_{0}^{+\infty}\left|\mathscr{H}_{\alpha}(\mathrm{h})(\lambda)\right|^{2} \lambda^{2} \mathrm{~d} \mu_{\alpha}(\lambda)
$$

adding (4.4) and (4.6), it follows that

$$
\|\operatorname{rh}\|_{2, \mu_{\alpha}}^{2}+\left\|\lambda \mathscr{H}_{\alpha}(h)\right\|_{2, \mu_{\alpha}}^{2} \geqslant 2 t^{2}\left[E_{\mu_{\alpha}}\left(|h|^{2}\right)+E_{\mu_{\alpha}}\left(\left|\mathscr{H}_{\alpha}(h)\right|^{2}\right)-2 \ln \left(\mathrm{t}^{2 \alpha+2}\right)\right] .
$$


Applying Theorem 3.5, we obtain

$$
\begin{aligned}
\|\mathrm{rh}\|_{2, \mu_{\alpha}}^{2}+\left\|\lambda \mathscr{H}_{\alpha}(\mathrm{h})\right\|_{2, \mu_{\alpha}}^{2} & \geqslant 2 \mathrm{t}^{2}[(2 \alpha+2)(1-2 \ln 2)-2(2 \alpha+2) \ln \mathrm{t}] \\
& =2 \mathrm{t}^{2}(2 \alpha+2)\left(1-\ln 2 \mathrm{t}^{2}\right) .
\end{aligned}
$$

In particular, for $t=\frac{1}{\sqrt{2}}$; we deduce that for every $h \in \mathrm{L}^{2}\left(d \mu_{\alpha}\right) ;\|h\|_{2, \mu_{\alpha}}=1$,

$$
\|\mathrm{rh}\|_{2, \mu_{\alpha}}^{2}+\left\|\lambda \mathscr{H}_{\alpha}(\mathrm{h})\right\|_{2, \mu_{\alpha}}^{2} \geqslant 2 \alpha+2 .
$$

Let $f \in L^{2}\left(d \mu_{\alpha}\right)$, replacing $h$ by $\frac{f}{\|f\|_{2, \mu_{\alpha}}^{2}}$ in relation (4.6), we claim that for every $f \in \mathrm{L}^{2}\left(\mathrm{~d} \mu_{\alpha}\right)$

$$
\|\mathrm{rf}\|_{2, \mu_{\alpha}}^{2}+\left\|\lambda \mathscr{H}_{\alpha}(\mathrm{f})\right\|_{2, \mu_{\alpha}}^{2} \geqslant(2 \alpha+2)\|f\|_{2, \mu_{\alpha}}^{2} .
$$

On the other hand, for $f \in L^{2}\left(d \mu_{\alpha}\right)$ and $t>0$, we denote by $f_{t}$ the dilated of $f$ defined by

$$
f_{t}(r)=f(t r)
$$

then, $f_{t}$ belongs to $L^{2}\left(d \mu_{\alpha}\right)$ and we have

$$
\begin{aligned}
\left\|f_{t}\right\|_{2, \mu_{\alpha}}^{2} & =\int_{0}^{+\infty}\left|f_{t}(r)\right|^{2} d \mu_{\alpha}(r) \\
& =\frac{1}{t^{2 \alpha+2}} \int_{0}^{+\infty}|f(r)|^{2} d \mu_{\alpha}(r) \\
& =\frac{1}{t^{2 \alpha+2}}\|f\|_{2, \mu_{\alpha}}^{2} .
\end{aligned}
$$

Moreover

$$
\begin{aligned}
\left\|r f_{t}\right\|_{2, \mu_{\alpha}}^{2} & =\int_{0}^{+\infty} r^{2}\left|f_{t}(r)\right|^{2} d \mu_{\alpha}(r) \\
& =\frac{1}{t^{2 \alpha+4}}\|r f\|_{2, \mu_{\alpha}}^{2},
\end{aligned}
$$

and

$$
\begin{aligned}
\left\|\lambda \mathscr{H}_{\alpha}\left(f_{\mathrm{t}}\right)\right\|_{2, \mu_{\alpha}}^{2} & =\int_{0}^{+\infty} \lambda^{2}\left|\mathscr{H}_{\alpha}\left(f_{\mathrm{t}}\right)(\lambda)\right|^{2} \mathrm{~d} \mu_{\alpha}(\lambda) \\
\mathscr{H}_{\alpha}\left(f_{\mathrm{t}}\right)(\lambda) & =\int_{0}^{+\infty} f_{\mathrm{t}}(x) j_{\alpha}(\lambda x) \mathrm{d} \mu_{\alpha}(x) \\
& =\frac{1}{\mathrm{t}^{2 \alpha+2}} \mathscr{H}_{\alpha}(\mathrm{f})\left(\frac{\lambda}{\mathrm{t}}\right) .
\end{aligned}
$$

Then

$$
\left\|\lambda \mathscr{H}_{\alpha}\left(f_{t}\right)\right\|_{2, \mu_{\alpha}}^{2}=\frac{1}{t^{2 \alpha}}\left\|\lambda \mathscr{H}_{\alpha}(f)\right\|_{2, \mu_{\alpha}}^{2} .
$$


Now, we assume that $\|\mathrm{rf}\|_{2, \mu_{\alpha}}$ and $\left\|\lambda \mathscr{H}_{\alpha}(f)\right\|_{2, \mu_{\alpha}}$ are both non zero and finite, hence the same holds for $f_{t}, t>0$ and from relation (4.7), we have

$$
\left\|\mathrm{rf}_{\mathrm{t}}\right\|_{2, \mu_{\alpha}}^{2}+\left\|\lambda \mathscr{H}_{\alpha}\left(f_{\mathrm{t}}\right)\right\|_{2, \mu_{\alpha}}^{2} \geqslant(2 \alpha+2)\left\|f_{\mathrm{t}}\right\|_{2, \mu_{\alpha}}^{2} .
$$

Then, by relations (4.8), (4.9) and (4.12), we get for every $t>0$

$$
\frac{1}{\mathrm{t}^{2 \alpha+4}}\|\mathrm{rff}\|_{2, \mu_{\alpha}}^{2}+\frac{1}{\mathrm{t}^{2 \alpha}}\left\|\lambda \mathscr{H}_{\alpha}(\mathrm{f})\right\|_{2, \mu_{\alpha}}^{2} \geqslant(2 \alpha+2) \frac{1}{\mathrm{t}^{2 \alpha+2}}\|\mathrm{f}\|_{2, \mu_{\alpha}}^{2}
$$

or

In particular for

$$
\frac{1}{\mathrm{t}^{2}}\|\mathrm{rf}\|_{2, \mu_{\alpha}}^{2}+\mathrm{t}^{2}\left\|\lambda \mathscr{H}_{\alpha}(\mathrm{f})\right\|_{2, \mu_{\alpha}}^{2} \geqslant(2 \alpha+2)\|\mathrm{f}\|_{2, \mu_{\alpha}}^{2} .
$$

$$
t=t_{0}=\sqrt{\frac{\|r f\|_{2, \mu_{\alpha}}}{\left\|\lambda \mathscr{H}_{\alpha}(f)\right\|_{2, \mu_{\alpha}}}} .
$$

We obtain

$$
\begin{gathered}
\left\|\lambda \lambda \mathscr{H}_{\alpha}(\mathrm{f})\right\|_{2, \mu_{\alpha}}\|\mathrm{rf}\|_{2, \mu_{\alpha}} \geqslant(\alpha+1)\|\mathrm{f}\|_{2, \mu_{\alpha}}^{2} \cdot \\
\text { Received: June 2014. Accepted: March } 2015 .
\end{gathered}
$$

\section{References}

[1] W. Beckner, Inequalities in Fourier analysis, Ann. of Math., 102 (1975) 159-182.

[2] W. Beckner, Pitt's inequality and the uncertainty principle, Proc. Amer. Math. Soc., (1995) 1897-1905.

[3] A. Beurling, The collected works of Arne Beurling, Birkhäuser, Vol.1-2, Boston 1989.

[4] I. Bialynicki-Birula, Entropic uncertainty relations in quantum mechanics, quantum probability and applications II, Eds. L. Accardi and W. Von Waldenfels, Lecture Notes in Mathematics, 1136 (1985) 90-103.

[5] A. Bonami, B. Demange, and P. Jaming, Hermite functions and uncertainty priciples for the Fourier and the widowed Fourier transforms, Rev. Mat. Iberoamericana, 19 (2003) 23-55.

[6] M.G. Cowling and J. F. Price, Generalizations of Heisenberg inequality in Harmonic analysis, (Cortona, 1982), Lecture Notes in Math., 992 (1983) 443-449.

[7] A. Erdélyi et al., Tables of integral transforms, Mc Graw-Hill Book Compagny., Vol.2, New York 1954.

[8] A. Erdélyi , Asymptotic expansions, Dover publications, New-York 1956. 
[9] A. Fitouhi, Inégalité de Babenko et inégalité logarithmique de Sobolev pour l'opérateur de Bessel, C. R. Acad. Sci. Paris, t. 305, Srie I, (1987) 877-880.

[10] G. B. Folland, Real analysis modern thechniques and their applications, Pure and Applied Mathematics, John Wiley and Sons, New York 1984.

[11] G. B. Folland and A. Sitaram, The uncertainty principle: a mathematical survey, J. Fourier Anal. Appl., 3 (1997) 207-238.

[12] G. H. Hardy, A theorem concerning Fourier transform, J. London. Math. Soc., 8 (1933) 227231.

[13] I. I. Hirschman, A note on entropy, Amer. J. Math., 79, 1 (1957) 152-156.

[14] I. I. Hirschman, Variation diminishing Hankel transforms, J. Anal. Math., Vol. 8, No. 01, (1960) 307-336.

[15] N. N. Lebedev, Special Functions and Their Applications, Dover publications, New-York 1972.

[16] G. W. Morgan, A note on Fourier transforms, J. London. Math. Soc., 9 (1934) 178-192.

[17] M. Rösler and M. Voit, An uncertaintly principle for the Hankel transforms, American Mathematical Society, 127 (1) (1999), 183-194.

[18] C. Shanon, A mathematical theory of communication, Bell System Tech. J., 27 (1948/1949), $379-423$ and $623-656$.

[19] K. Trimèche, Transformation intégrale de Weyl et théorème de Paley-Wiener associés à un opérateur différentiel singulier sur $(0,+\infty), J$. Math. Pures Appl., 60 (1981) 51-98.

[20] K. Trimèche, Inversion of the Lions transmutation operator using genaralized wavelets, Appl. Comput. Harmon. Anal., 4 (1997) 97-112.

[21] Vu Kim Tuan, Uncertainty principle for the Hankel transform. Integral Transforms Spec. Funct. Vol. 18, Issue 5 (2007), 369-381.

[22] G. N. Watson, A treatise on the theory of Bessel functions, Cambridge univ. Press., 2nd ed., Cambridge 1959. 